\title{
A Green's function for diffraction by a rational wedge
}

\author{
BY A. D. RAWLINS \\ Department of Mathematics and Statistics, Brunel University, \\ Uxbridge, Middlesex $U B 83 P H$
}

(Received 14 October 1987; revised 18 March 1988)

\begin{abstract}
In this paper we derive an expression for the point source Green's function for the reduced wave equation, valid in an angular sector whose angle is equal to a rational multiple of $\pi$. This Green's function can be used to find new expressions for the field produced by the diffraction of a spherical wave by a wedge whose angle can be expressed as a rational multiple of $\pi$. The expressions obtained will be in the form of source terms and real integrals representing the diffracted field. The general result obtained is used to derive a new representation for the solution of the problem of diffraction by a mixed hard-soft half plane.
\end{abstract}

\section{Introduction}

In this work we shall give new solutions to various problems of diffraction of a spherical acoustic wave by a wedge whose angle can be expressed as a rational multiple of $\pi$. The solutions, corresponding to various soft (Dirichlet) and/or hard (Neumann) boundary conditions on the wedge faces, is given in terms of geometrical acoustic source terms, and real integrals representing the diffracted field, for all positions of source and receiver.

In acoustics such a field representation is useful for physical interpretation and analytical calculations. Besides the problems of diffraction by a soft wedge or a hard wedge, we include in our consideration the field produced by diffraction of the spherical source wave by a hard-soft wedge, that is, a wedge, one face of which is rigid, the other soft. This type of boundary value problem has applications in noise barrier design (see Rawlins [9]), and in underwater acoustic problems connected with sound propagation in coastal channels (see Jacobson et al. [4, 5]).

In $1896 \mathrm{~A}$. Sommerfeld obtained an exact closed form solution to the problem of plane wave diffraction by a half plane. He used the method of images on Riemann surfaces corresponding to multivalued solutions of the reduced wave equation, and indicated how this method could be used to obtain exact closed form solution for the problem of diffraction by a wedge. The method of constructing the required manyvalued solution of the wave equation was simplified by Sommerfeld in a subsequent paper [13]. Subsequently Carslaw [2] replaced the image method by a direct construction of the solution which yields simpler formulae for various types of wave sources. These solutions had been obtained independently by Macdonald [6, 7] by summing the Fourier series representation of the Green's function. Other methods of 
obtaining some or all of the principal results have since been put forward by various authors, including Bromwich [1], Whipple [14], Garnir[3] and Oberhettinger [8]. The last two authors give extensive bibliographies.

Recently Rawlins [10,11] derived some new representations for the Green's function for plane and cylindrical wave diffraction by a rational wedge. The result for the cylindrical wave Green's function was based on the plane wave Green's function. Here we derive in a different, more direct, and simpler manner the Green's function for a spherical point source. The method used here can be applied without difficulty to deal with more general types of sources, in particular the plane wave and cylindrical wave source already considered.

In $\S 2$ we shall give the geometry of the physical diffraction problems. In $\S 3$ we shall define a periodic Green's function as the solution of a periodic boundary value problem for a wedge of open angle $2 \alpha$. By means of this periodic Green's function we show in §4 that one can obtain expressions for the Green's function for Dirichlet, Neumann or mixed Dirichlet-Neumann boundary value problems for a wedge of open angle $\alpha$. In $\S 5$ we give a general complex integral representation for the periodic Green's function. This complex integral representation for the periodic Green's function is not new. It has appeared in various guises in the papers already cited. In $\$ 6$ we show how this Green's function can be reduced to source terms and real integrals representing the diffracted waves for the special case of a rational wedge $\alpha=p \pi / q$ ( $p$ and $q$ positive integers). Finally in $\S 7$ we give an application in the form of a new solution for the problem of acoustic diffraction of a spherical wave by a hard-soft half plane.

\section{Geometry of diffraction problems}

We shall be interested in finding solution to diffraction problems in wedge shaped regions. To be specific, we shall consider acoustic diffraction by time harmonic waves (with harmonic time variation $e^{i \omega t}$ assumed, but not shown explicitly, in the rest of the paper).

We shall work in Euclidean space of three dimensions with cylindrical polar coordinates $(r, \theta, z)$. In this space we shall assume there is a wedge of open angle $\alpha$ with faces defined by the planes $\theta=0$ and $\theta=\alpha$. For $\alpha=0$ the wedge disappears; for $\alpha=\pi$ it becomes a half space. For $\alpha=2 \pi$ the wedge becomes a semi-infinite plane whose upper and lower faces are considered as distinct.

In physical problems $\alpha$ is necessarily not greater than $2 \pi$. However, in the mathematical analysis that follows we shall be dealing with functions that make mathematical sense for all real $\alpha$. Therefore it is of interest to consider wedge angles $\alpha$ which are virtual with opening $\alpha>2 \pi$; their existence is purely mathematical.

We shall consider two points $P \equiv(r, \theta, z)$ and $P_{0} \equiv\left(r_{0}, \theta_{0}, z_{0}\right)$ which both lie within the open angle region $0<\theta<\alpha$, and let

$$
\begin{aligned}
& R\left(P, P_{0}\right)=\left(r_{0}^{2}+r^{2}+\left(z-z_{0}\right)^{2}-2 r r_{0} \cos \left(\theta-\theta_{0}\right)\right)^{\frac{1}{2}}, \\
& D\left(P, P_{0}\right)=\left(\left(r+r_{0}\right)^{2}+\left(z-z_{0}\right)^{2}\right)^{\frac{1}{2}} .
\end{aligned}
$$

$R\left(P, P_{0}\right)$ is the shortest distance (in the Euclidean plane) between $P_{0}$ and $P$; and $D\left(P, P_{0}\right)$ is the shortest distance of the broken line from $P_{0}$ to the edge of the wedge and thence to $P$.

We shall assume that a time harmonic point wave source is situated at $P_{0}$. 


\section{Periodic boundary value problem defining a Green's function}

We shall define a periodic Green's function for the reduced wave equation operator $\nabla^{2}+k^{2}$ (where $k=\omega / c, c$ being the velocity of sound) by means of a periodic boundary value problem posed on a wedge, real or virtual, of open angle $2 \alpha$.

We shall denote this periodic Green's function by $G_{\alpha}\left(r, \theta, z ; r_{0}, \theta_{0}, z_{0} ; k\right)$ or $G_{\alpha}\left(P, P_{0} ; k\right)$ for an angular opening $2 \alpha . G_{\alpha}\left(P, P_{0} ; k\right)$, which is a function of $P$ and $P_{0}$, is defined uniquely by the following periodic boundary value problem.

(a) It is a solution of

$$
\left(\nabla^{2}+k^{2}\right) G_{\alpha}\left(P, P_{0} ; k\right)=\delta\left(P, P_{0}\right) \quad(0<r<\infty, 0<\theta<2 \alpha,-\infty<z<\infty) .
$$

(b) It satisfies the periodic boundary conditions

$$
\begin{aligned}
G_{\alpha}\left(r, 0, z ; r_{0}, \theta_{0}, z_{0} ; k\right) & =G_{\alpha}\left(r, 2 \alpha, z ; r_{0}, \theta_{0}, z_{0} ; k\right), \\
\frac{\partial G_{\alpha}}{\partial \theta}\left(r, 0, z ; r_{0}, \theta_{0}, z_{0} ; k\right) & =\frac{\partial G_{\alpha}}{\partial \theta}\left(r, 2 \alpha, z ; r_{0}, \theta_{0}, z_{0} ; k\right) .
\end{aligned}
$$

(c) It satisfies the Sommerfeld radiation condition

$$
\lim _{R\left(P, P_{0}\right) \rightarrow \infty} R\left(P, P_{0}\right)\left(\frac{\partial G_{\alpha}\left(P, P_{0} ; k\right)}{\partial R\left(P, P_{0}\right)}+i k G_{\alpha}\left(P, P_{0} ; k\right)\right)=0 .
$$

(d) It satisfies the edge condition

$$
G_{\alpha}\left(P, P_{0} ; k\right)=O(1) \text { and }\left|\operatorname{grad} G_{\alpha}\left(P, P_{0} ; k\right)\right|=o(1 / r) \text { as } r \rightarrow 0 .
$$

We remark that it follows from the above properties that the Green's function will be symmetric, i.e.

$$
G_{\alpha}\left(P, P_{0} ; k\right)=G_{\alpha}\left(P_{0}, P ; k\right)
$$

Once the above periodic Green's function has been obtained, it is a simple matter to obtain Green's functions for acoustically hard, soft or mixed hard-soft boundary value problems for wedges of half this open angle.

\section{Green's functions for hard and soft boundaries for a wedge of open angle $\alpha$}

To simplify notation in this section we shall let the periodic Green's function $G_{\alpha}\left(r, \theta, z ; r_{0}, \theta_{0}, z_{0} ; k\right)$ be denoted by $G_{\alpha}(\theta)$ or equivalently $G_{\alpha}\left(\theta_{0}\right)$. Then it is not difficult to show, using the fact that $G_{\alpha}(2 n \alpha+\theta)=G_{\alpha}(\theta)$ where $n$ is any integer, that: the Green's function $G_{\alpha}^{8}\left(P, P_{0} ; k\right)$ for a soft wedge of open angle $\alpha$ is given by

$$
G_{\alpha}^{8}\left(P, P_{0} ; k\right)=G_{\alpha}(\theta)-G_{\alpha}(2 \alpha-\theta) ;
$$

the Green's function $G_{\alpha}^{h}\left(P, P_{0} ; k\right)$ for a hard wedge of open angle $\alpha$ is given by

$$
G_{\alpha}^{h}\left(P, P_{0} ; k\right)=G_{\alpha}(\theta)+G_{\alpha}(2 \alpha-\theta)
$$

In the same way it can be shown that

$$
G_{\alpha}^{s, h}\left(P, P_{0} ; k\right)=G_{2 \alpha}(\theta)+G_{2 \alpha}(2 \alpha-\theta)-G_{2 \alpha}(2 \alpha+\theta)-G_{2 \alpha}(4 \alpha-\theta)
$$

is the appropriate Green's function for the wedge which is soft on the face $\theta=0$ and hard on the face $\theta=\alpha$. The corresponding Green's function for a hard boundary on the face $\theta=0$ and a soft boundary on the face $\theta=\alpha$ is given by

$$
G_{\alpha}^{h, 8}\left(P, P_{0} ; k\right)=G_{2 \alpha}(\theta)-G_{2 \alpha}(2 \alpha-\theta)-G_{9_{\sim}}(2 \alpha+\theta)+G_{\rho_{\sim}}(4 \alpha-\theta) .
$$


For $\pi<\alpha \leqslant 2 \pi$ and $\pi / 2<\alpha \leqslant 2 \pi$ the functions $G_{\alpha}(\theta)$ and $G_{2 \alpha}(\theta)$ respectively, mathematically relate to a virtual wedge angle, which explains the necessity to consider such wedges.

\section{A complex integral representation of the periodic Green's function}

A complex integral representation for the periodic Green's function defined in $\S 3$ for $\delta\left(P, P_{0}\right)=-4 \pi \delta\left(\left|P-P_{0}\right|\right)$ has been shown by Carslaw and others [3] to be given by

$$
G_{\alpha}\left(r, \theta, z ; r_{0}, \theta_{0}, z_{0} ; k\right)=\frac{1}{2 i \alpha} \int_{\mathscr{G}} \mathscr{G}(\zeta) \frac{\sin (\zeta \pi / \alpha) d \zeta}{\cos (\zeta \pi / \alpha)-\cos \left(\left(\theta-\theta_{0}\right) \pi / \alpha\right)}
$$

where

$$
\mathscr{G}(\zeta)=\frac{e^{-\imath k \mathscr{R}(\zeta)}}{\mathscr{R}(\zeta)}, \mathscr{R}(\zeta)=\left(r^{2}+r_{0}^{2}+\left(z-z_{0}\right)^{2}-2 r r_{0} \cos \zeta\right)^{\frac{1}{2}}
$$

The square root is defined uniquely by $\mathscr{R}(0)=D\left(P, P_{0}\right)$, so that $\mathscr{R}(\zeta)$ is analytic everywhere except along branch cuts that extend vertically up and down from branch points above and below the real axis, respectively. These branch points are located at $2 \pi l \pm i \beta$, where $\beta=\cosh ^{-1}\left\{\left(r^{2}+r_{0}^{2}+\left(z-z_{0}\right)^{2}\right) /\left(2 r r_{0}\right)\right\}$, and $l$ is any integer : see Figure 1. In the chosen cut plane $-\pi / 2 \leqslant \arg \mathscr{R}(\zeta) \leqslant \pi / 2$. The contour of integration $\mathscr{C}$ in the expression (1) is such that the starting point is given by $c_{1}+i \infty$ and the termination point is given by $c_{2}+i \infty$, where $-\pi \leqslant c_{1} \leqslant 0, \pi \leqslant c_{2} \leqslant 2 \pi$; and the contour lies between the branch points $\zeta= \pm i \beta$ and does not intersect any of the other branch cuts which run parallel to the imaginary axis: see Figure 1.

We shall now use the complex integral representation (1) to obtain a different representation for the special case of $\alpha$ being a rational multiple of $\pi$, i.e. $\alpha=p \pi / q$, where $p$ and $q$ are positive integers. This new representation will be in terms of point source terms and real integrals. From the results we have already given in $\S 4$ this means we can represent the acoustic field scattered by a soft, hard, or mixed soft-hard wedge with an open angle $\alpha=p \pi / q$ in terms of point sources and real integrals representing the diffracted field.

\section{Green's function for a rational wedge}

If the angle is $\alpha=p \pi / q$, where $p$ and $q$ are positive integers, the expression (1) becomes

$$
G_{p \pi / q}\left(P, P_{0} ; k\right)=\frac{1}{2 i p \pi} \int_{\mathscr{G}} \mathscr{G}(\zeta) \frac{q \sin (\zeta q / p) d \zeta}{\cos (\zeta q / p)-\cos \left(\left(\theta-\theta_{0}\right) q / p\right)} .
$$

By using the identity

$$
\frac{q \sin (\zeta p / q)}{\cos (\zeta p / q)-\cos \left(\left(\theta-\theta_{0}\right) p / q\right)}=\sum_{m=0}^{q-1} \frac{\sin (\zeta / p)}{\cos (\zeta / p)-\cos \left(\left(\theta-\theta_{0}\right) / p+2 \pi m / q\right)}
$$

(see Rawlins[10]) we can rewrite (3) in the form

where

$$
\begin{aligned}
& G_{p \pi / q}\left(P, P_{0} ; k\right)=\sum_{m=0}^{q-1} I_{p}\left(\theta-\theta_{0}+2 \pi m p / q\right), \\
& I_{p}(\psi)=\frac{1}{2 \pi i p} \int_{\mathscr{C}} \mathscr{G}(\zeta) \frac{\sin (\zeta / p) d \zeta}{\cos (\zeta / p)-\cos (\psi / p)} .
\end{aligned}
$$




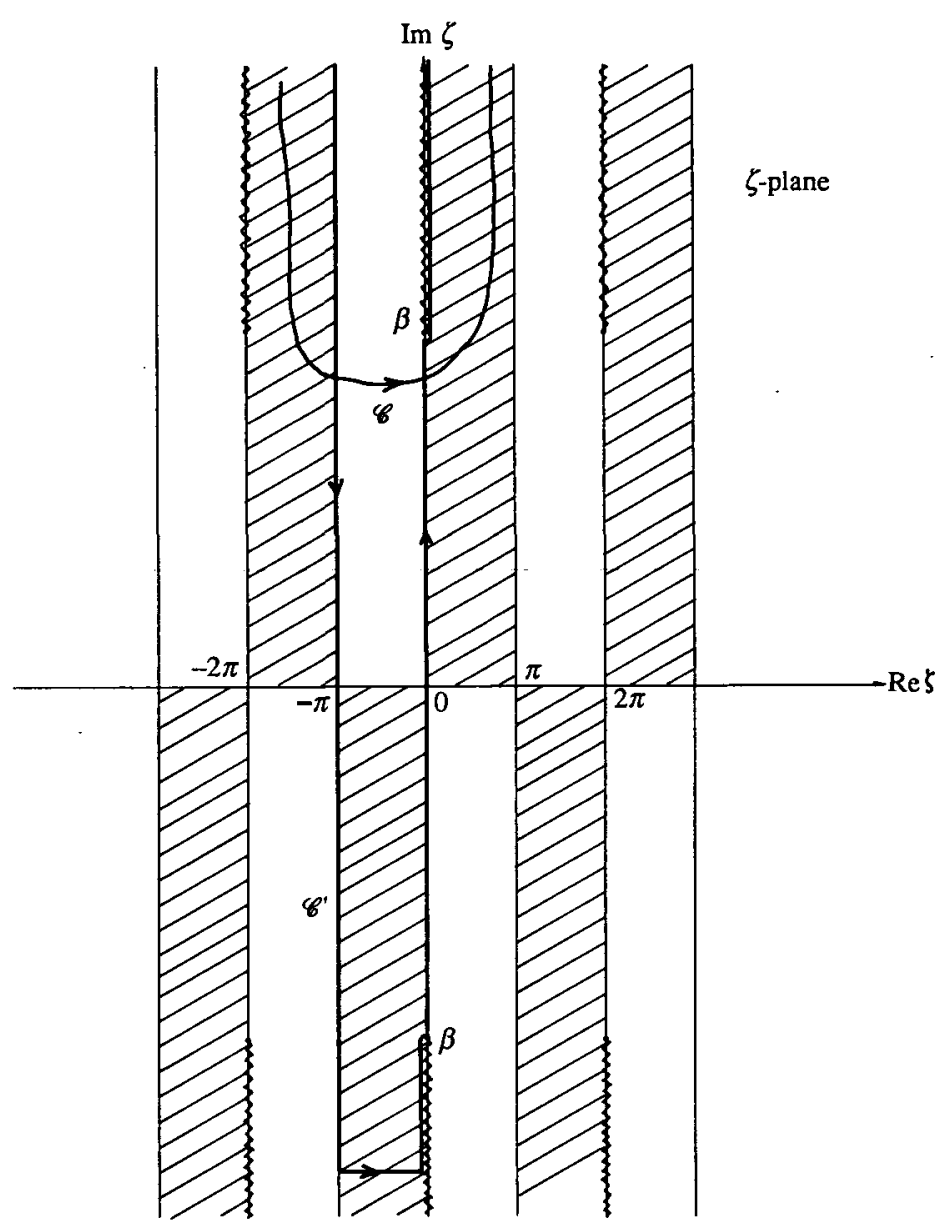

Fig. 1. Diagram of the paths of integration in the $\zeta$-plane. The shaded regions show where the integral (6) is uniformly convergent. The wavy lines are the branch cuts for the function $\mathscr{G}(\zeta)$.

We now distort the path of integration $\mathscr{C}$ in the integral (6), so that it takes up the new path $\mathscr{C}^{\prime}$, as shown in Figure 1. The new path of integration $\mathscr{C}^{\prime}$ lies along regions where the integral is uniformly convergent. In distorting the contour $\mathscr{C}$ to take up the contour $\mathscr{C}^{\prime}$ the line $0 \leqslant \operatorname{Re} \zeta \leqslant \pi, \operatorname{Im} \zeta=0$, is crossed, and therefore if any zeros of $\cos (\zeta / p)-\cos (\psi / p)$, (viz $\zeta= \pm(\psi+2 \pi p N)$ where $N$ is an integer) are captured then they will give rise to pole contributions. Thus

$$
\begin{aligned}
I_{p}(\psi)= & \sum_{N} H[\pi-|\psi+2 \pi p N|] \mathscr{G}(\psi+2 \pi p N) \\
& -\frac{1}{2 \pi i p}\left\{\int_{-\beta}^{\beta}+\int_{\beta}^{\infty}+\int_{-\infty}^{-\beta}\right\} \mathscr{G}(i y) \frac{\sinh (y / p) d y}{\cosh (y / p)-\cos (\psi / p)} \\
& +\frac{1}{2 \pi i p} \int_{-\infty}^{\infty} \mathscr{G}(\pi+i y) \frac{\sin ((\pi+i y) / p) i d y}{\cos ((i y+\pi) / p)-\cos (\psi / p)} \\
& +\lim _{\rho \rightarrow \infty} \frac{1}{2 \pi i p} \int_{0}^{\pi} \mathscr{G}(x-i \rho) \frac{\sin ((x-i \rho) / p) d x}{\cos ((x-i \rho) / p-\cos (\psi / p)}
\end{aligned}
$$


where

$$
H[x]=\left\{\begin{array}{l}
0 \text { for } x<0 \\
\frac{1}{2} \text { for } x=1 \\
1 \text { for } x>1
\end{array}\right.
$$

is the Heaviside step function, and where the summation is performed for all integer values of $N$ which satisfy the inequality $-\pi \leqslant \psi+2 \pi p N \leqslant \pi$.

In the above expression the first integral has had its limits of integration broken up, because the path traverses branch cuts. It can be shown, using the appropriately defined values of $\mathscr{G}(i y)$ on the branch cuts, that this whole term vanishes because the integrand is odd in $y$. The last integral in the above expression also gives zero contribution because $\lim _{\rho \rightarrow \infty}|\mathscr{G}(x-i \rho)| \rightarrow 0$ for $0 \leqslant x \leqslant \pi$. Thus

$$
\begin{aligned}
& I_{p}(\psi)=\sum_{N} H[\pi-|\psi+2 \pi p N|] \mathscr{G}(\psi+2 \pi p N) \\
&+\frac{1}{2 \pi i p} \int_{-\infty}^{\infty} \mathscr{G}(\pi+i y) \frac{\sin ((\pi+i y) / p) i d y}{\cos ((i y+\pi) / p)-\cos (\psi / p)}
\end{aligned}
$$

By using the identity (see Rawlins [10])

$$
\begin{aligned}
& \frac{(1 / p) \sin (\zeta / p)}{\cos (\zeta / p)-\cos (\psi / p)} \\
& \quad=\frac{a_{p-1}(\psi) \sin (\zeta / p)+\sum_{n-0}^{p-2} a_{n}(\psi)\{\sin (\zeta(p-n) / p)-\sin (\zeta(p-2-n) / p)\}}{\cos \zeta-\cos \psi},
\end{aligned}
$$

where $a_{n}(\psi)=\sin ((n+1) \psi / p) /\{p \sin (\psi / p)\}$, in the expression (7) we have

$$
\begin{aligned}
I_{p}(\psi)= & \sum_{N} H[\pi-|\psi+2 \pi p N|] \mathscr{G}(\psi+2 \pi p N) \\
& -\frac{1}{2 \pi} a_{p-1}(\psi) \int_{-\infty}^{\infty} \frac{\mathscr{G}(\pi+i y) \sin ((\pi+i y) / p) d y}{\cosh y+\cos \psi} \\
& -\frac{1}{2 \pi} \sum_{n=0}^{p-2} a_{n}(\psi) \int_{-\infty}^{\infty} \frac{\mathscr{G}(\pi+i y)}{\cosh y+\cos \psi} \\
& \quad \times\left\{\sin \left((\pi+i y) \frac{(p-n)}{p}\right)-\sin \left((\pi+i y) \frac{(p-2-n)}{p}\right)\right\} d y .
\end{aligned}
$$

Now by using the fact that $\mathscr{G}(\pi+i y)=\mathscr{G}(\pi-i y)$ we can write the above expression as

$$
\begin{aligned}
I_{p}(\psi)= & \sum_{N} H[\pi-|\psi+2 \pi p N|] \mathscr{G}(\psi+2 \pi p N) \\
& -\frac{1}{\pi} a_{p-1}(\psi) \sin \left(\frac{\pi}{p}\right) \int_{0}^{\infty} \frac{\mathscr{G}(\pi-i y) \cosh (y / p) d y}{\cosh y+\cos \psi} \\
& -\frac{1}{\pi} \sum_{n=1}^{p-2}\left\{a_{n}(\psi) \sin \left(\pi \frac{(p-n)}{p}\right) \int_{0}^{\infty} \frac{\mathscr{G}(\pi-i y) \cosh (y(p-n) / p) d y}{\cosh y+\cos \psi}\right. \\
& \left.-a_{n-1}(\psi) \sin \left(\pi \frac{(p-1-n)}{p}\right) \int_{0}^{\infty} \frac{\mathscr{G}(\pi-i y) \cosh (y(p-1-n) / p) d y}{\cosh y+\cos \psi}\right\} .
\end{aligned}
$$


Thus finally on substituting (8) into (5) we get

$$
\begin{aligned}
G_{p \pi / q}\left(P, P_{0} ; k\right)= & \sum_{m=0}^{q-1} \sum_{N} H\left[\pi-\left|\theta-\theta_{0}+2 \pi m p / q+2 \pi p N\right|\right] \mathscr{G}\left(\theta-\theta_{0}+2 \pi m p / q+2 \pi p N\right) \\
& -\frac{1}{p \pi} \sum_{m=0}^{q-1} \frac{\sin \left(\theta-\theta_{0}+2 \pi m p / q\right) \sin (\pi / p)}{\sin \left(\left(\theta-\theta_{0}+2 \pi m p / q\right) / p\right)} \\
& \times \int_{0}^{\infty} \frac{\cosh (y / p) \mathscr{G}(\pi-i y) d y}{\cosh y+\cos \left(\theta-\theta_{0}+2 \pi m p / q\right)} \\
& -\frac{1}{p \pi} \sum_{m=0}^{q-1} \sum_{n=1}^{p-2}\left\{\frac{\sin \left((n+1)\left(\theta-\theta_{0}+2 \pi m p / q\right) / p\right) \sin (n \pi / p)}{\sin \left(\left(\theta-\theta_{0}+2 \pi m p / q\right) / p\right)}\right. \\
& -\frac{\sin \left(n\left(\theta-\theta_{0}+2 \pi m p / q\right) / p\right) \sin ((n+1) \pi / p)}{\sin \left(\left(\theta-\theta_{0}+2 \pi m p / q\right) / p\right)} \\
\times \int_{0}^{\infty} \frac{\cosh ((p-n) y / p) \mathscr{G}(\pi-i y) d y}{\cosh y+\cos \left(\theta-\theta_{0}+2 \pi m p / q\right)} &
\end{aligned}
$$

where the summation over $N$ is for all integer values of $N$ which can make the argument of the Heaviside step function non-negative, and where $\mathscr{G}(\zeta)$ is given by equation (2).

As a specific application we shall give the solution to the problem of acoustic diffraction of a spherical sound wave by a hard-soft half plane.

\section{Diffraction by a hard-soft half plane}

In terms of the Green's function the solution $U_{h, s}$ for the problem of diffraction of the spherical wave $\mathscr{G}\left(\theta-\theta_{0}\right)$ by a hard-soft half plane is given by

$$
\begin{aligned}
U_{h, s}=G_{4 \pi}\left(r, \theta, z, r_{0}, \theta_{0}, z_{0} ; k\right)+G_{4 \pi}\left(r, \theta, z, r_{0},-\theta_{0}, z_{0} ; k\right) \\
\quad-G_{4 \pi}\left(r, \theta, z, r_{0}, 4 \pi-\theta_{0}, z_{0} ; k\right)-G_{4 \pi}\left(r, \theta, z, r_{0}, 4 \pi+\theta_{0}, z_{0} ; k\right)
\end{aligned}
$$

By using the expression (9) with $p=4, q=1$, it is not difficult to show that

$$
\begin{aligned}
U_{h, s}= & H\left[\pi-\left|\theta-\theta_{0}\right|\right] \mathscr{G}\left(\theta-\theta_{0}\right)+H\left[\pi-\left|\theta+\theta_{0}\right|\right] \mathscr{G}\left(\theta+\theta_{0}\right) \\
& -H\left[\pi-\left|\theta+\theta_{0}-4 \pi\right|\right] \mathscr{G}\left(\theta+\theta_{0}\right)-\frac{1}{\sqrt{ } 2 \pi} \cos \frac{1}{4}\left(\theta-\theta_{0}\right) \int_{0}^{\infty} \frac{\cosh \frac{3}{4} t \mathscr{G}(\pi-i t)}{\cosh t+\cos \left(\theta-\theta_{0}\right)} d t \\
& -\frac{1}{\sqrt{ } 2 \pi} \cos \frac{1}{4}\left(\theta+\theta_{0}\right) \int_{0}^{\infty} \frac{\cosh \frac{3}{4} t \mathscr{G}(\pi-i t)}{\cosh t+\cos \left(\theta+\theta_{0}\right)} d t \\
& +\frac{1}{\sqrt{ } 2 \pi} \cos \frac{1}{4}\left(\theta-\theta_{0}\right)\left(1-2 \cos \frac{1}{2}\left(\theta-\theta_{0}\right)\right) \int_{0}^{\infty} \frac{\cosh \frac{1}{4} t \mathscr{G}(\pi-i t)}{\cosh t+\cos \left(\theta-\theta_{0}\right)} d t \\
& +\frac{1}{\sqrt{ } 2 \pi} \cos \frac{1}{4}\left(\theta+\theta_{0}\right)\left(1-2 \cos \frac{1}{2}\left(\theta+\theta_{0}\right)\right) \int_{0}^{\infty} \frac{\cosh \frac{1}{4} t \mathscr{G}(\pi-i t)}{\cosh t+\cos \left(\theta+\theta_{0}\right)} d t
\end{aligned}
$$


The first term of the above expression represents the incident field which will only exist in the region $\pi \geqslant\left|\theta-\theta_{0}\right|$. The second term represents the reflected field from the rigid upper face of the half plane, which will only be present for $\pi \geqslant\left|\theta+\theta_{0}\right|$. The third term represents the reflected wave from the soft lower face of the half plane, which will only be present for $\pi \geqslant\left|\theta+\theta_{0}-4 \pi\right|$. The remaining integrals represent the field diffracted by the edge of the half plane.

In conclusion we remark that if we replace $\mathscr{G}(\zeta)$ in the expression (9) by $e^{i k r \cos \zeta}$ or $H_{0}^{(2)}(k \mathscr{R}(\zeta))$ we get the appropriate Green's function for a plane or cylindrical wave source, respectively. If we replace $\mathscr{G}(\zeta)$ by $1 / \mathscr{R}(\zeta)$, we obtain the Green's function for Laplace's equation, which has applications in incompressible fluid mechanics and electrostatics. Inversion of this problem will give the Green's function for a lens problem as well. The author hopes to pursue this aspect in a later paper.

\section{REFERENCES}

[1] T. J. L'A Bromwich. Diffraction of waves by a wedge. Proc. London Math. Soc. (2) 14 (1915), $450-463$.

[2] H. S. Carslaw. Diffraction of waves by a wedge of any angle. Proc. London Math. Soc. (2) 18 (1919), 291-307.

[3] H. G. Garnir. Fonctions de Green de l'opérateur métaharmonique pour les problèmes de Dirichlet et de Neumann posés dans un angle ou un dièdre. Bull. Soc. Roy. Sci. Liège 21 (1952), 119-140, 207-231.

[4] M. I. Jacobson and J.G. Clark. Refraction/reflected ray transmission in a divergent channel. J. Acoust. Soc. Amer. 41 (1967), 167-176.

[5] M. I. JACOBSON and J. T. W ARFIELD. Surface reflected/bottom-reflected ray transmission in a divergent channel. $J$. Acoust. Soc. Amer. 43 (1968), 15-24.

[6] H. M. Macdonald. Electric Waves (Cambridge University Press, 1902).

[7] H. M. Macdonald. A class of diffraction problems. Proc. London Math Soc. (2) 14 (1915), 410-427.

[8] F. Oberbettinger. On the diffraction and reflection of waves and pulses by wedges and corners. J. Res. Nat. Bur. Standards 61 (1958), 343-365.

[9] A. D. Rawlins. The solution of a mixed boundary value problem in the theory of diffraction by a semi-infinite plane. Proc. Roy. Soc. London Ser. A 346 (1975), 469-484.

[10] A. D. Rawlins. Plane wave diffraction by a rational wedge. Proc. Roy. Soc. London Ser. A 411 (1987), 265-283.

[11] A. D. Rawlins. Cylindrical wave diffraction by a rational wedge. Proc. Roy. Soc. London Ser. A 411 (1987), 285-303.

[12] A. Sommerfeld. Mathematische Theorie der Diffraktion. Math. Ann. 47 (1896), 317-374.

[13] A. Sommerfeld. Über verzweigte Potentiale in Raum. Proc. London Math. Soc. (1) 28 (1897), $395-429$.

[14] F. J. WhIPPLE. Diffraction by a wedge and kindred problems. Proc. London Math. Soc. (2) 16 (1917), 94-111. 